International Mathematical Forum, 1, 2006, no. 32, 1595-1597

\title{
Construction of singular surfaces over a finite field
}

\author{
E. Ballico ${ }^{1}$ \\ Dept. of Mathematics, University of Trento \\ 38050 Povo (TN), Italy \\ ballico@science.unitn.it
}

\begin{abstract}
Fix a prime power $q$ and integers $m \geq 2, \sigma>0, x>0, g \geq 0$, $d \geq m \sigma+1$ such that $q \geq(\delta-1) \delta^{3}$, where $\delta:=d^{3}+3 d x+4 x+2 g-2$. Let $C \subset \mathbf{P}^{3}$ be a smooth degree $x$ curve defined over $\mathbb{F}_{q}$ such that $h^{1}\left(\mathbf{P}^{3}, \mathcal{I}_{C}(\sigma-1)\right)=$ $h^{1}\left(C, \mathcal{O}_{C}(\sigma-2)\right)=0$ and $p_{a}(C)=g$. Here we prove the existence of a degree $d$ surface $X \subseteq \mathbf{P}^{3}$ defined over $\mathbb{F}_{q}$, such that $\operatorname{Sing}(X)=C$ and $X$ has ordinary multiplicity $m$ along $C$, i.e. for every $P \in C\left(\bar{F}_{q}\right)$ the tangent cone of $X$ at $P$ is reduced and it is the union of $m$ distinct planes containing the tangent line of $C$ at $P$.
\end{abstract}

Mathematics Subject Classification: 14J25; 14J70; 14N05; 12E20; 14B05; 14B25

Keywords: singular hypersurface; singular surface; surfaces over a finite field

\section{Singular SURfaces OVER A Finite FIELD}

Many existence theorems are easy over an algebraically closed base field $K$. When $K=\bar{F}_{p}$ there is a large $p$-power $q$ such that a solution may be defined over $\mathbb{F}_{q}$ with $q$ not too large. Here we prove the existence of certain degree $d$ surfaces $X \subset \mathbf{P}^{3}$ with as singular locus a prescribed smooth curve and with a prescribed multiplicity at each point of it and defined over $\mathbb{F}_{q}$. More precisely, we prove the following result.

Theorem 1. Fix a prime power $q$ and integers $m \geq 2, \sigma>0, x>0, g \geq 0$, $d \geq m \sigma+1$ such that $q \geq(\delta-1) \delta^{3}$, where $\delta:=d^{3}+3 d x+4 x+2 g-2$. Let $C \subset \mathbf{P}^{3}$ be a smooth degree $x$ curve defined over $\mathbb{F}_{q}$ such that $h^{1}\left(\mathbf{P}^{3}, \mathcal{I}_{C}(\sigma-1)\right)=$ $h^{1}\left(C, \mathcal{O}_{C}(\sigma-2)\right)=0$ and $p_{a}(C)=g$. Then there exists a degree $d$ surface $X \subseteq \mathbf{P}^{3}$ defined over $\mathbb{F}_{q}$, such that $\operatorname{Sing}(X)=C$ and $X$ has ordinary multiplicity $m$ along $C$, i.e. for every $P \in C\left(\bar{F}_{q}\right)$ the tangent cone of $X$ at $P$ is reduced and it is the union of $m$ distinct planes containing the tangent line of $C$ at $P$.

By Castelnuovo-Mumford's lemma the homogeneous ideal of $C$ is generated by forms of degree at most $\sigma$. In the statement of Theorem 1 we do not assume that $C$ is connected. When $C$ is connected we may take $\sigma=x-1$, and all cases in which we cannot take $\sigma=x-2$ are classified ([3]).

\footnotetext{
${ }^{1}$ The author was partially supported by MIUR and GNSAGA of INdAM (Italy)
} 
Lemma 1. Let $C \subset \mathbf{P}^{3}$ be an integral curve such that

$$
h^{1}\left(\mathbf{P}^{3}, \mathcal{I}_{C}(\sigma-1)\right)=h^{1}\left(C, \mathcal{O}_{C}(\sigma-2)\right)=0 .
$$

Fix an integer $t \geq \sigma+1, P \in C_{\text {reg }}$ and a tangent vector $\tau$ of $\mathbf{P}^{3}$ at $P$. Then $h^{1}\left(\mathbf{P}^{3}, \mathcal{I}_{C \cup \tau}(z)\right)=h^{2}\left(\mathbf{P}^{3}, \mathcal{I}_{C \cup \tau}(z-1)\right)=0$ for all $z \geq \sigma$, the homogeneous ideal of $C \cup \tau$ is generated by forms of degree at most $\sigma+1$ and there exists a degree $t$ surface containing $C \cup \tau$, but smooth at $P$.

Proof. If $\tau$ is tangent to $C$, then $\tau \subset C$ and hence there is nothing to prove. Thus we may assume that $\tau$ is not tangent to $C$ at $P$. By Castelnuovo-Mumford's lemma it is sufficient to prove $h^{1}\left(\mathbf{P}^{3}, \mathcal{I}_{C \cup \tau}(\sigma)\right)=h^{2}\left(\mathbf{P}^{3}, \mathcal{I}_{C \cup \tau}(\sigma-1)\right)=0$; indeed, note that since the tangent space of $C \cup \tau$ is two-dimensional and $C \cup \tau$ is schemetheoretically cut out by degree $t$ surfaces, at least one degree $t$ surface containing $C \cup \tau$ must be smooth at $P$. Since $\operatorname{dim}(\tau)=0$, we have $h^{2}\left(\mathbf{P}^{3}, \mathcal{I}_{C \cup \tau}(\sigma-1)\right)=$ $h^{1}\left(C \cup \tau, \mathcal{O}_{C \cup \tau}(\sigma-1)\right)=h^{1}\left(C, \mathcal{O}_{C}(\sigma-1)\right)=0$. Let $H \subset \mathbf{P}^{3}$ be a plane containing $\tau$ and transversal to $C$ at $P$. Since $C$ is the residual scheme of $C \cup \tau$ with respect to $H$, there is the following exact sequence:

$$
0 \rightarrow \mathcal{I}_{C}(\sigma-1) \rightarrow \mathcal{I}_{C \cup \tau}(\sigma) \rightarrow \mathcal{I}_{(C \cap \tau) \cap H}(\sigma) \rightarrow 0
$$

Hence it is sufficient to prove $h^{1}\left(H, \mathcal{I}_{(C \cap \tau) \cap H}(\sigma)\right)=0$. We know that $h^{1}\left(H, \mathcal{I}_{C \cap H, H}(\sigma-\right.$ $1))=0$. Let $R \subset H$ be the line spanned by $\tau$. Since the homogeneous ideal of $C \cap H$ in $H$ is generated by forms of degree at most $\sigma$, we have length $(C \cap R) \leq \sigma$. Thus length $((C \cup \tau) \cap R)) \leq \sigma+1$. Since the residual scheme of $(C \cap \tau) \cap H$ with respect to $R$ is $(C \cap H) \backslash(C \cap R)$ we have an exact sequence

$$
0 \rightarrow \mathcal{I}_{(C \cap H) \backslash(C \cap R), H}(\sigma-1) \rightarrow \mathcal{I}_{(C \cup \tau) \cap H, H}(\sigma) \rightarrow \mathcal{I}_{C \cap R, R}(\sigma) \rightarrow 0
$$

We have $h^{1}\left(R, \mathcal{I}_{C \cap R, R}(\sigma)\right)=0$, because length $\left.((C \cup \tau) \cap R)\right) \leq \sigma+1$. We have $H^{1}\left(H, \mathcal{I}_{(C \cap H) \backslash(C \cap R), H}(\sigma-1)\right) \leq h^{1}\left(H, \mathcal{I}_{C \cap H, H}(\sigma-1)\right)=0$.

Remark 1. Let $X$ be an integral projective variety and $L, M \in \operatorname{Pic}(W)$. If $L$ is very ample and $M$ is spanned, then $L \otimes M$ is very ample.

Proof of Theorem 1. Let $N_{C}$ denote the normal sheaf of $C$ in $\mathbf{P}^{3}$. Since $C$ is smooth, $N_{C}$ is a rank 2 vector bundle with degree $2 g-2+4 x$. Let $w: W \rightarrow \mathbf{P}^{3}$ be the blowing-up of $C$. Set $E:=w^{-1}(C)$ and $\mathcal{O}_{W}(1):=w^{*}\left(\mathcal{O}_{\mathbf{P}^{3}}(1)\right)$. Hence $E$ and $\mathcal{O}_{W}(1)$ freely generate $\operatorname{Pic}(W)$. We have $\mathcal{O}_{W} \cdot E \cdot E=-x, \mathcal{O}_{W}(1) \cdot \mathcal{O}_{W}(1) \cdot E=0$, $\mathcal{O}_{W}(1)^{3}=1$ and $E^{3}=-4 x+2-2 g$ ([2], Prop. 6.7). For all integers $t, c$ set $\mathcal{L}_{t, c}:=\mathcal{O}_{W}(t)(-c E)$. Notice that $\mathcal{L}_{d, m}^{3}=d^{3}+3 d x+4 x+2 g-2=\delta$.

(a) Here we will check that $\mathcal{L}_{t, 1}$ is spanned for all $t \geq \sigma$. Fix $Q \in W$. First assume $Q \notin E$. Thus $w(Q) \notin C$. Since $t \geq \sigma$, there is a degree $t$ surface $A \subset \mathbf{P}^{3}$ containg $C$ and with $w(Q) \notin C$. The strict transform $A^{\prime}$ of $A$ in $W$ is an element of $\left|\mathcal{L}_{t, c}\right|$ for some integer $c \geq 1$. Hence $A^{\prime \prime}:=A^{\prime}+(c-1)(E) \in\left|\mathcal{L}_{t, 1}\right|$ and $Q \notin A^{\prime \prime}$. Now assume $Q \in E$. Thus $Q$ represents a tangent vector $\tau$ of $\mathbf{P}^{3}$ at $w(Q)$ not in the tangent line to $C$ at $P$. Since $C$ is scheme-theoretically cut out inside $\mathbf{P}^{3}$ by all degree $t$ hypersurfaces containing $C$, there is one such surface $B$ whose tangent plane at $w(Q)$ does not contain $\tau$. Use the strict transform of $B$ to show that $Q$ is not in the base locus of $\left|\mathcal{L}_{t, 1}\right|$. 
(b) Here we will check that $\mathcal{L}_{t, 1}$ is very ample for all $t \geq \sigma+1$. It is sufficient to check that $h^{0}\left(W, \mathcal{L}_{t, 1}(-Z)\right)=h^{0}\left(W, \mathcal{L}_{t, 1}\right)-2$ for all length two zero-dimensional schemes $Z \subset W$. We need to distinguish 6 cases:

(i) $Z$ is reduced, say $Z=\left\{Q, Q^{\prime}\right\}$, with $Q \notin E$ and $Q^{\prime} \notin E$;

(ii) $Z$ is reduced, say $Z=\left\{Q, Q^{\prime}\right\}$, with $Q \notin E$ and $Q^{\prime} \in E$;

(iii) $Z$ is reduced and $Z \subset E$;

(iv) $Z$ is not reduced and $Q:=Z_{\text {red }} \notin E$.

(v) $Z$ is not reduced, $Q:=Z_{\text {red }} \in E$ and $Z$ is not in the tangent plane to $E$ at $Q$;

(vi) $Z$ is not reduced and $Z \subset E$.

We will only check case (vi). By part (a) it is sufficient to check that for all $P \in C$ and all tangent vectors $\tau$ of $\mathbf{P}^{3}$ at $P$ there is a degree $t$ surface $U \subset \mathbf{P}^{3}$ such that $C \cup \tau \subset U$ and $U$ is smooth at $P$. This is proved in Lemma 1. By Remark 1 our assumption on the integer $d$ implies that $\mathcal{L}_{d, m}$ is very ample. By [1], Th. 1, our assumption on $q$ and the equality $\delta=\mathcal{L}_{d, m}^{3}$ implies the existence of a smooth $\Sigma \in\left|\mathcal{L}_{d, m}\right|$ defined over $\mathbb{F}_{q}$. The surface $w(\Sigma)$ is a solution of Theorem 1 .

\section{REFERENCES}

[1] E. Ballico, An effective Bertini theorem over finite fields, Adv. Geom. 3 (2003), no. 4, 361-363.

[2] W. Fulton, Intersection Theory, Springer, Berlin, 1984.

[3] L. Gruson, R. Lazarsfeld and Ch. Peskine, On a theorem of Castelnuovo and the equations defining space curves, Invent. Math. 72 (1983), no. 3, 491-506.

Received: January 23, 2006 